\title{
Intrinsic Contact Angle of Aqueous Phases at Membranes and Vesicles
}

\author{
Halim Kusumaatmaja, Yanhong Li, Rumiana Dimova, and Reinhard Lipowsky \\ Theory \& Bio-Systems, Max Planck Institute of Colloids and Interfaces, 14424 Potsdam, Germany
}

(Received 25 August 2009; published 2 December 2009)

\begin{abstract}
Biomimetic membranes exposed to several aqueous phases are studied theoretically. Specific examples are provided by lipid vesicles and phase separating polymer solutions. The theory reveals the existence of an intrinsic contact angle, $\theta_{\text {in }}$, between the membranes and the aqueous phases. An explicit relation is derived by which this intrinsic angle can be determined from the effective contact angles as measured by optical microscopy. A detailed comparison between theory and experiment provides strong evidence that the intrinsic contact angle $\theta_{\text {in }}$ represents a material parameter, in contrast to the effective contact angles.
\end{abstract}

PACS numbers: 87.16.D-, 68.05. $-\mathrm{n}$, 68.08.Bc

Recently, novel soft matter systems consisting of lipid vesicles and two species of water-soluble polymers such as dextran and polyethylene glycol (PEG) have been introduced and studied experimentally [1-3]. When the polymer concentrations exceed a few weight percent, the aqueous solution undergoes phase separation, and the lipid membranes are exposed to different aqueous phases. This process leads to shape transformations of the vesicles [2] and to wetting transitions of the aqueous phases [3].

Phase separation within the vesicles can be induced by osmotic deflation, i.e., by adding osmotically active particles to the exterior solution. The resulting osmotic unbalance leads to water permeation through the vesicle membranes and to a reduced volume of the vesicles. Since the polymers cannot permeate the membrane, the polymer concentration is increased, and the aqueous solution forms two separate phases, $\alpha$ and $\beta$, within the vesicles. For the dextran/PEG solutions in [1-3], the phases $\alpha$ and $\beta$ correspond to the PEG-rich and dextranrich phase, respectively.

Phase separation within a single vesicle proceeds via several stages as observed in the optical microscope [4]. First, many small droplets are formed within the vesicle. These droplets then grow and coalesce until the interior compartment of the vesicle contains only two large droplets, one $\alpha$ and one $\beta$ droplet [5]. As a result, a single vesicle typically attains a state as shown schematically in Fig. 1(a). In such a state, both liquid phases $\alpha$ and $\beta$ are in contact with the vesicle membrane corresponding to partial wetting of this membrane [3].

In this Letter, we present the first systematic theory for membranes and vesicles in contact with several aqueous phases. Our theory starts from the simple observation that a vesicle state as in Fig. 1(a) involves different types of surfaces. First, the two aqueous phases $\alpha$ and $\beta$ are separated by a fluid-fluid interface with interfacial tension $\Sigma_{\alpha \beta}$. The boundary of the $\alpha \beta$ interface along the vesicle membrane defines the contact line, at which all three liquid phases $\alpha, \beta$, and $\gamma$ are in close proximity. This contact line divides the membrane into two distinct segments, separating the $\alpha$ and $\beta$ phases from the exterior solution denoted by $\gamma$. In general, these two segments are found to experience two distinct mechanical tensions, $\Sigma_{\alpha \gamma}$ and $\Sigma_{\beta \gamma}$.

When viewed with optical resolution, the membrane shape exhibits a kink along the contact line as in Fig. 1(a). One can then deduce three effective contact angles $\theta_{\alpha}, \theta_{\beta}$, and $\theta_{\gamma}$ with $\theta_{\alpha}+\theta_{\beta}+\theta_{\gamma}=2 \pi$. These contact angles are related to the three tensions $\Sigma_{\alpha \beta}, \Sigma_{\alpha \gamma}$, and $\Sigma_{\beta \gamma}$ via the classical Neumann equations [6] for the force balance along the contact line. However, if the membrane shape had a kink that persisted to smaller length scales, its bending energy would become infinite. Therefore, when viewed with suboptical resolution, the membrane should be smoothly curved, which implies the existence of an intrinsic contact angle $\theta_{\text {in }}$ as in Fig. 1(b).

We will show below that the intrinsic angle $\theta_{\text {in }}$ is related to the effective contact angles via the simple relation

$$
\cos \theta_{\text {in }}=\frac{\sin \theta_{\alpha}-\sin \theta_{\beta}}{\sin \theta_{\gamma}}=\frac{\sin \theta_{\alpha}-\sin \theta_{\beta}}{\sin \left(2 \pi-\theta_{\alpha}-\theta_{\beta}\right)}
$$

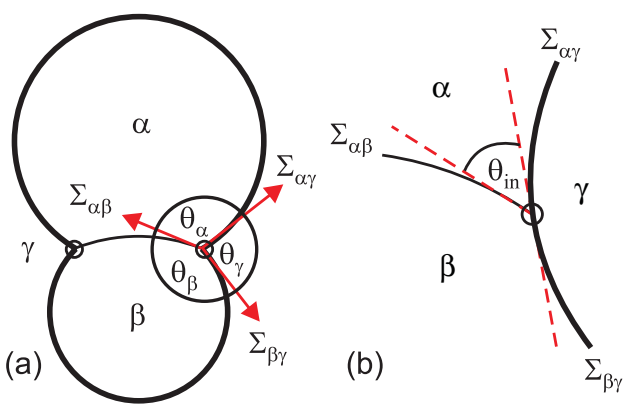

FIG. 1 (color). (a) Cross-section of a vesicle enclosing one $\alpha$ (top) and one $\beta$ (bottom) droplet suspended in the exterior solution $\gamma$. When viewed with optical resolution, the vesicle shape exhibits a sharp kink along the contact line $(\odot)$ and can be characterized by three effective contact angles $\theta_{\alpha}, \theta_{\beta}$, and $\theta_{\gamma}$ with $\theta_{\alpha}+\theta_{\beta}+\theta_{\gamma}=2 \pi$. These contact angles are related to the three tensions $\Sigma_{\alpha \beta}, \Sigma_{\alpha \gamma}$, and $\Sigma_{\beta \gamma}$ via the force balance along the contact line; and (b) Enlarged view close to the contact line: Intrinsic contact angle $\theta_{\text {in }}$ between the two planes that are tangential to the $\alpha \beta$ interface and to the smoothly curved vesicle membrane, respectively. 
up to corrections arising from the contact line tension. Thus, the numerical value of the intrinsic contact angle $\theta_{\text {in }}$ can be determined via relation (1) using the effective contact angles $\theta_{\alpha}, \theta_{\beta}$, and $\theta_{\gamma}$ as measured by optical microscopy. Furthermore, a detailed comparison between theory and experiment provides strong evidence that the intrinsic contact angle $\theta_{\text {in }}$ represents a material parameter, whereas the tensions $\Sigma_{\alpha \gamma}$ and $\Sigma_{\beta \gamma}$ as well as the effective contact angles depend on the vesicle geometry.

Theoretical description.-To proceed, let us consider the various contributions to the total energy $E$ of a vesicle as in Fig. 1(a). First, this energy contains two terms that depend on the volumes $V_{\alpha}$ and $V_{\beta}$ of the $\alpha$ and $\beta$ phases and on the pressure differences $P_{\gamma}-P_{i}$ with $i \equiv \alpha, \beta$ between the exterior phase $\gamma$ and the two interior phases. Second, the $\alpha \beta$ interface with area $A_{\alpha \beta}$ and the two membrane segments with areas $A_{\alpha \gamma}$ and $A_{\beta \gamma}$ contribute three surface terms. Third, the contact line provides an energetic contribution proportional to its length $L_{\alpha \beta \gamma}$ and to its line tension $\lambda$. Finally, the vesicle energy contains the bending energies $E_{\mathrm{be}, i}$ of the two membrane segments. Thus, the total energy has the form

$$
E=\sum_{i=\alpha, \beta}\left[E_{\mathrm{dr}, i}+E_{\mathrm{be}, i}\right]+\Sigma_{\alpha \beta} A_{\alpha \beta}+\lambda L_{\alpha \beta \gamma}
$$

with the "droplet" energies $E_{\mathrm{dr}, i} \equiv\left(P_{\gamma}-P_{i}\right) V_{i}+\Sigma_{i \gamma} A_{i \gamma}$ and the bending energies

$$
E_{\mathrm{be}, i}=\int d A_{i \gamma}\left[2 \kappa_{i \gamma}\left(M-M_{i \gamma}\right)^{2}+\kappa_{G, i \gamma} G\right],
$$

which depend on the mean curvature $M$ and the Gaussian curvature $G$ of the membrane as well as on the bending rigidities $\kappa_{i \gamma}$, the spontaneous mean curvatures $M_{i \gamma}$, and the Gaussian curvature moduli $\kappa_{G, i \gamma}$. The energy $E$ as in (2) and (3) is similar to the energy of vesicles with several intramembrane domains as studied previously in $[7,8]$. The corresponding theoretical predictions in [7] have been fully confirmed by several experimental studies [9-11].

In order to simplify our description, we will now assume (i) that the two membrane segments have the same bending rigidity $\kappa=\kappa_{\alpha \gamma}=\kappa_{\beta \gamma}$, (ii) that the spontaneous mean curvatures $M_{i \gamma}$ can be neglected, and (iii) that the two membrane segments have the same Gaussian curvature moduli $\kappa_{G}=\kappa_{G, \alpha \gamma}=\kappa_{G, \beta \gamma}$, which implies that the Gaussian curvature moduli do not affect the vesicle shape because of the Gauss-Bonnet theorem [12].

Vesicles enclosing two liquid droplets as in Fig. 1(a) are observed to be axisymmetric. The corresponding symmetry axis will be chosen to be the $z$ axis, the distance from this axis will be denoted by $\rho$. The vesicle shape is then uniquely described by its one-dimensional contour $(z(t), \rho(t))$ as shown in Fig. 2. The contour parameter $t$ varies over two fixed intervals, and the integrals over the areas $A_{\alpha \gamma}$ and $A_{\beta \gamma}$ now become $t$ integrals over $t_{0}=0 \leq$ $t \leq t_{1}$ and $t_{1} \leq t \leq t_{2}$. Thus, the values $t_{0}=0, t_{1}$, and $t_{2}$ correspond to the north pole, the position of the contact line, and the south pole, respectively, and the contact line length $L_{\alpha \beta \gamma}=2 \pi \rho\left(t_{1}\right)$. Two additional quantities that play an important role in the theory are the arc length $s(t)$ and the tilt angle $\psi(t)$, see Fig. 2. Using this parametrization, the two volume terms in (2) become

$$
\begin{aligned}
\sum_{i=\alpha, \beta}\left(P_{\gamma}-P_{i}\right) V_{i}= & \sum_{i=\alpha, \beta}\left(P_{\gamma}-P_{i}\right) \int_{i} d t s^{\prime} \rho^{2} \sin \psi \\
& +\left(P_{\alpha}-P_{\beta}\right) \Delta V_{\beta}
\end{aligned}
$$

where the prime indicates a derivative with respect to $t$ and $\Delta V_{\beta}$ denotes the volume of the spherical cap as indicated by the gray area in Fig. 2. Using the Laplace equation for the $\alpha \beta$ interface, a combination of the $\Delta V_{\beta}$ term in (4) with the term $\Sigma_{\alpha \beta} A_{\alpha \beta}$ in (2) leads to

$$
\left(P_{\alpha}-P_{\beta}\right) \Delta V_{\beta}+\Sigma_{\alpha \beta} A_{\alpha \beta}=\frac{2 \pi}{3} \frac{\left(1-\cos ^{3} \theta\right)}{\sin ^{2} \theta} \Sigma_{\alpha \beta} \rho\left(t_{1}\right)^{2}
$$

where we have introduced the tangent angle $\theta$ between the $\alpha \beta$ interface and the horizontal plane, see Fig. 2.

The total energy $E$ of the vesicle now has the form

$$
\frac{E}{2 \pi}=\sum_{i=\alpha, \beta} \int_{i} d t \mathcal{L}_{i}+\lambda \rho\left(t_{1}\right)+\frac{1}{3} \frac{\left(1-\cos ^{3} \theta\right)}{\sin ^{2} \theta} \Sigma_{\alpha \beta} \rho\left(t_{1}\right)^{2}
$$

with the "Lagrange functions"

$$
\begin{aligned}
\mathcal{L}_{i} \equiv & \frac{\kappa}{2} \rho s^{\prime}\left(\frac{\psi^{\prime}}{s^{\prime}}+\frac{\sin \psi}{\rho}\right)^{2}+\Sigma_{i \gamma} \rho s^{\prime} \\
& +\frac{1}{2}\left(P_{\gamma}-P_{i}\right) \rho^{2} s^{\prime} \sin \psi+\Upsilon\left(\rho^{\prime}-s^{\prime} \cos \psi\right)
\end{aligned}
$$

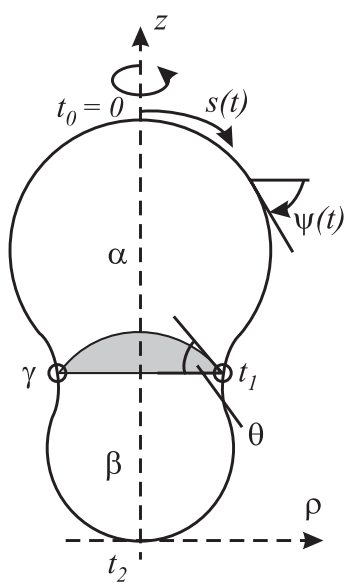

FIG. 2. Axisymmetric vesicle containing one $\alpha$ and one $\beta$ droplet. The coordinate along the symmetry axis is denoted by $z$, the distance from this axis by $\rho$, the arc length by $s$, and the local tilt angle by $\psi$. All of these variables depend on the contour parameter $t$ with $t_{0}=0 \leq t \leq t_{2}$. The contact line is located at $t=t_{1}$, the gray area corresponds to the spherical cap with volume $\Delta V_{\beta}$ and tangent angle $\theta$. 
and the Lagrange multiplier function $Y$ used to ensure the geometrical relation $\rho^{\prime}=s^{\prime} \cos \psi$. The energy $E$ in (6) has the same form as the one used in [7], except for the new boundary term $\sim \Sigma_{\alpha \beta} \rho\left(t_{1}\right)^{2}$ arising from the $\alpha \beta$ interface.

The first variation of the energy $E$ in (6) leads to the same "Euler-Lagrange" or shape equations as derived previously in [7]. In addition, this variation implies the boundary conditions

$$
\begin{gathered}
\dot{\psi}\left(s_{1}\right)_{\beta}=\dot{\psi}\left(s_{1}\right)_{\alpha} \quad \text { with } \quad s_{1} \equiv s\left(t_{1}\right), \\
\ddot{\psi}\left(s_{1}\right)_{\beta}-\ddot{\psi}\left(s_{1}\right)_{\alpha}=\frac{\Sigma_{\alpha \beta}}{\kappa} \sin \theta_{\text {in }}+\frac{\lambda}{\kappa \rho\left(s_{1}\right)} \sin \psi\left(s_{1}\right),
\end{gathered}
$$

and

$$
\Sigma_{\beta \gamma}-\Sigma_{\alpha \gamma}=\Sigma_{\alpha \beta} \cos \theta_{\text {in }}+\frac{\lambda}{\rho\left(s_{1}\right)} \cos \psi\left(s_{1}\right)
$$

along the contact line, where the overdots now denote derivatives with respect to $s$ and the geometrical relation $\theta_{\text {in }}=\psi\left(s_{1}\right)-\theta$ for the intrinsic contact angle $\theta_{\text {in }}$ has been used. The first condition (8) represents the torque balance along the contact line [13]. The second condition (9) and the third condition (10) describe the balance between the force components perpendicular and parallel, respectively, to the plane tangential to the smoothly curved membrane.

The terms in the boundary conditions (9) and (10) that are proportional to the contact line tension $\lambda$ are expected to have a negligible effect on the intrinsic contact angle if the contact line distance $\rho\left(s_{1}\right) \gg \rho_{*} \equiv \lambda / \Sigma_{\alpha \beta}$. The tension $\Sigma_{\alpha \beta}$ of the $\alpha \beta$ interface has been measured to be of the order of $10 \mu \mathrm{N} / \mathrm{m}$ [4]. For the multicomponent membranes studied in [9-11], the line tension of the domain boundaries was found to vary between $10^{-12}$ and $10^{-14} \mathrm{~N}$. If the contact line tension $\lambda$ considered here had similar values, one would have $1 \mathrm{~nm} \lesssim \rho_{*} \lesssim 100 \mathrm{~nm}$ and $\rho\left(s_{1}\right) \gg \rho_{*}$ since $\rho\left(s_{1}\right)$ exceeded a couple of micrometers for all vesicles studied experimentally. Therefore, the corrections arising from the line tension will now be ignored.

The force balance Eq. (10) without the $\lambda$ term can be deduced from two general properties: (i) a smoothly curved membrane along the contact line and (ii) constant mechanical tensions $\Sigma_{i \gamma}$ within the two membrane segments. Therefore, the relation (10) is not restricted to axisymmetric shapes but is valid for any shape of the vesicle.

Strong tension regime.-An experimentally relevant limit is the case, in which the interfacial energy as given by $\Sigma_{\alpha \beta} A_{\alpha \beta}$ is much larger than the bending rigidity $\kappa$, i.e., if the radius $R_{\alpha \beta} \equiv \sqrt{A_{\alpha \beta} / \pi} \gg \sqrt{\kappa / \Sigma_{\alpha \beta}} \equiv R_{*}$. In this limit, the Euler-Lagrange or shape equations reduce to the Laplace equation for liquid droplets, and the membrane segments then attain the shapes of spherical caps, apart from a small region close to the three-phase contact line. The width of this latter region is of the order of $R_{*}$. For the membrane/polymer systems studied here, the bending rigidity $\kappa \simeq 10^{-19} \mathrm{~J}$ and the interfacial tension $\Sigma_{\alpha \beta} \simeq$
$10 \mu \mathrm{N} / \mathrm{m}$ which implies $R_{*} \simeq 100 \mathrm{~nm}$. Since this latter scale is below optical resolution, the vesicle membrane appears to have a kink along the contact line and the effective contact angles $\theta_{\alpha}, \theta_{\beta}$, and $\theta_{\gamma}$ as defined in Fig. 1(a) can be directly deduced from the optical micrographs. These contact angles satisfy the relations $\Sigma_{\alpha \gamma} / \Sigma_{\alpha \beta}=\sin \theta_{\beta} / \sin \theta_{\gamma}$ and $\Sigma_{\beta \gamma} / \Sigma_{\alpha \beta}=\sin \theta_{\alpha} / \sin \theta_{\gamma}$ as follows from the Neumann equations [6]. Inserting these relations into the boundary condition (10) without the $\lambda$ term leads to (1).

Measurements of contact angles.-Aqueous solutions composed of $2.10 \mathrm{wt} \%$ PEG $(8 \mathrm{~kg} / \mathrm{mol})$ and $7.44 \mathrm{wt} \%$ dextran $(500 \mathrm{~kg} / \mathrm{mol})$ were encapsulated within giant vesicles. At room temperature, the solutions prepared in this way are homogeneous. In order to induce phase separation into dextran- and PEG-rich phases, the polymer concentration was raised above the binodal curve by deflation, i.e., by exposing the vesicles to a hypertonic medium. In order to balance the resulting osmotic pressure, water was released from the vesicles. The increase in polymer concentration then induced phase separation within the vesicles. The membranes themselves consisted of $95.9 \mathrm{~mol} \%$ 1,2-dioleoyl$s n$-glycero-3-phosphocholine (DOPC), $4.0 \mathrm{~mol} \% G_{M 1}$ ganglioside (brain, ovine-ammonium salt), and $0.1 \mathrm{~mol} \%$ 1, 2-dipalmitoyl- $s n$-glycero-3-phosphoethanolamine- $N$ (lissamine rhodamine B sulfonyl) (DPPE-rhod); for further details, see [4].

An ensemble of vesicles prepared in the same batch and deflated in the same chamber was studied in detail.

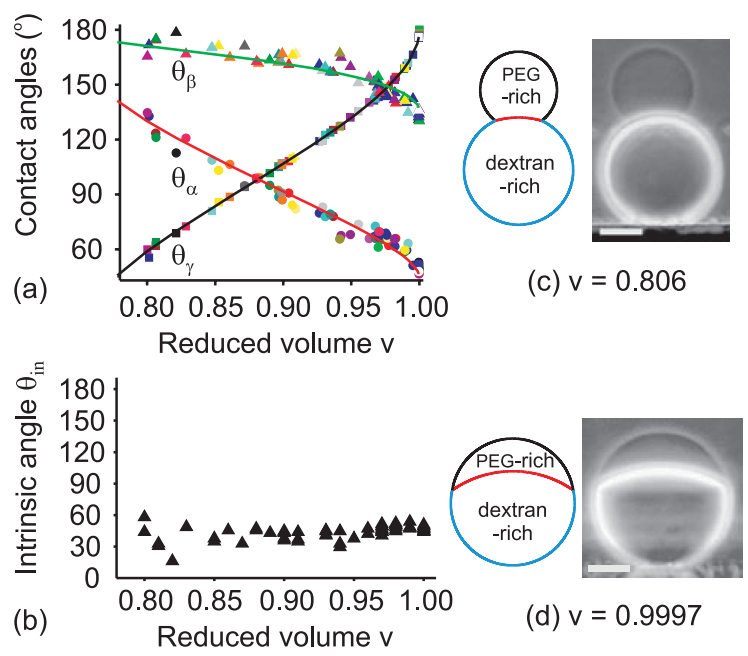

FIG. 3 (color). (a) Effective and (b) intrinsic contact angles in degrees as a function of the reduced volume $v$ for volume ratio $V_{\alpha} / V_{\beta}=0.33 \pm 0.06$ and essentially fixed polymer composition of the $\alpha$ and $\beta$ droplets. In (a), the effective contact angles $\theta_{\alpha}, \theta_{\beta}$, and $\theta_{\gamma}$ as measured for 63 different vesicles are shown in different colors (scattered symbols) and compared with the theoretical calculations (full lines); and (c),(d) Comparison between calculated vesicle shapes (left) and phase contrast vesicle images (right) for two different values of reduced volume $v$. The scale bars are $20 \mu \mathrm{m}$. 
Deflation was induced by raising the osmolarity of the exterior solution from 22 to $33 \mathrm{Osm} / \mathrm{m}^{3}$. The resulting phase separation process led to the formation of lipid aggregates [3] and membrane tubes [4], and to the fission of small vesicles. Thus, both the volume and the apparent area of the vesicles were reduced during deflation. We then waited $21 \mathrm{~h}$ to obtain osmotically balanced vesicles. After this equilibration, all $\alpha$ and $\beta$ droplets exerted almost the same osmotic pressures $P_{\alpha} \simeq P_{\gamma}$ and $P_{\beta} \simeq P_{\gamma}$ onto the membranes [14]. This mechanical equilibrium implies that all $\alpha$ and $\beta$ droplets contained almost the same particle number density of osmotically active particles. Furthermore, within each vesicle, the $\alpha$ and $\beta$ droplet were in chemical equilibrium as well. Therefore, all $\alpha$ droplets had essentially the same polymer composition corresponding to the binodal curve of the phase diagram, and likewise for all $\beta$ droplets.

In order to compare theory and experiment, we selected 63 vesicles with volume ratio $V_{\alpha} / V_{\beta}=0.33 \pm 0.06$ and measured the effective contact angles via vesicle image fitting. These contact angles are shown in Fig. 3(a) as a function of the reduced volume $v \equiv V_{\text {ve }} /[(4 \pi / 3) \times$ $\left.\left(A_{\mathrm{ve}} / 4 \pi\right)^{3 / 2}\right]$ with $V_{\mathrm{ve}} \equiv V_{\alpha}+V_{\beta}$ and $A_{\mathrm{ve}} \equiv A_{\alpha \gamma}+A_{\beta \gamma}$. From the dataset in Fig. 3(a), the intrinsic contact angle $\theta_{\text {in }}$ can be calculated using relation (1), see Fig. 3(b). We find that the intrinsic contact angle is roughly constant with $\cos \theta_{\text {in }}=0.714 \pm 0.075$. Our claim that the angle $\theta_{\text {in }}$ is a material property is further supported by micropipette experiments in [15].

Vesicle shapes in the spherical cap approximation.Apart from an overall length scale, the shape of the vesicle can be determined from the shape equations if the intrinsic contact angle $\theta_{\text {in }}$, the reduced volume $v$, and the volume ratio $V_{\alpha} / V_{\beta}$ are known. Within the spherical cap approximation, one then obtains explicit expressions for $v$ and $V_{\alpha} / V_{\beta}$ in terms of the effective contact angles and the tangent angle $\theta$. Using these expressions together with (1), we find excellent agreement between theory and experiment as illustrated in Figs. 3(c) and 3(d) for two different values of the reduced volume. For the theoretical calculations shown in Figs. 3(a), 3(c), and 3(d), we used the average values $\cos \theta_{\text {in }}=0.714$ and $V_{\alpha} / V_{\beta}=0.33$. When $v$ is considerably smaller than 1 as in Fig. 3(c), the vesicle buds to minimize the interfacial area between the $\alpha$ and $\beta$ droplets as will be described in more detail in [15].

Conclusions.-In summary, the requirement of a smoothly curved membrane along the contact line leads to the concept of an intrinsic contact angle $\theta_{\text {in }}$, see Fig. 1(b). For the membrane/polymer systems studied in [1-3] and in this Letter, the angle $\theta_{\text {in }}$ represents a "hidden variable" since it cannot be directly observed in the optical microscope, see Fig. 1(a) as well as Figs. 3(c) and 3(d). Our theory shows, however, that the angle $\theta_{\text {in }}$ is an essential property of these systems. First, it determines the boundary conditions (9) and (10) along the contact line. Second, the numerical value of $\theta_{\text {in }}$ can be calculated from the force balance relation (1), using the effective contact angles $\theta_{\alpha}$, $\theta_{\beta}$, and $\theta_{\gamma}$ as deduced from the optical micrographs, see Fig. 3(a). Third, for a given membrane/polymer system, the angle $\theta_{\text {in }}$ is found to be constant and independent of geometric parameters such as the reduced volume, see Fig. 3(b). We conclude that $\theta_{\text {in }}$ represents a material parameter in contrast to the effective contact angles, which strongly depend on the vesicle geometry as in Fig. 3(a). Therefore, the formation of coexisting phases $\alpha$ and $\beta$ within a vesicle affects the vesicle shape via two material parameters, the interfacial tension $\Sigma_{\alpha \beta}$ and the intrinsic contact angle $\theta_{\text {in }}$.

In future research, it will be of great interest to study the morphological diagrams of vesicles with two aqueous phases as a function of $\Sigma_{\alpha \beta}$ and $\theta_{\text {in }}$ and to identify shape transformations induced or modified by the presence of these two phases. Another open issue is provided by line tension effects, which become important for sufficiently small $\alpha \beta$ interfaces. Finally, phase separation within vesicles often leads to the formation of many thin membrane tubes [4], a process that remains to be elucidated.

[1] M. R. Helfrich et al., J. Am. Chem. Soc. 124, 13374 (2002).

[2] M. S. Long, A. S. Cans, and C. D. Keating, J. Am. Chem. Soc. 130, 756 (2008).

[3] Y.-H. Li, R. Lipowsky, and R. Dimova, J. Am. Chem. Soc. 130, 12252 (2008).

[4] Y.-H. Li, Ph.D. thesis, University of Potsdam, 2008; http:// nbn-resolving.de/urn:nbn:de:kobv:517-opus-29138.

[5] Vesicles with several buds enclosing $\alpha$ or $\beta$ droplets have also been observed but usually represent metastable states. In addition, complete budding can induce fission.

[6] J.S. Rowlinson and B. Widom, Molecular Theory of Capillarity (Clarendon Press, Oxford, 1989).

[7] F. Jülicher and R. Lipowsky, Phys. Rev. Lett. 70, 2964 (1993); Phys. Rev. E 53, 2670 (1996).

[8] E. Gutlederer, T. Gruhn, and R. Lipowsky, Soft Matter 5, 3303 (2009).

[9] T. Baumgart, S. T. Hess, and W. W. Webb, Nature (London) 425, 821 (2003); T. Baumgart, S. Das, W. W. Webb, and J. T. Jenkins, Biophys. J. 89, 1067 (2005).

[10] K. Bacia, P. Schwille, and T. Kurzchalia, Proc. Natl. Acad. Sci. U.S.A. 102, 3272 (2005).

[11] S. Semrau et al., Phys. Rev. Lett. 100, 088101 (2008).

[12] M.P. do Carmo, Differential Geometry of Curves and Surfaces (Prentice-Hall, Englewood Cliffs, 1976).

[13] The relations (8)-(10) can also be derived using the formalism in J.-B. Fournier, Soft Matter 3, 883 (2007).

[14] The pressure differences $\left(P_{i}-P_{\gamma}\right)$ or Laplace pressures are of the order of $\Sigma_{\alpha \beta} M \sim 1 \mathrm{~N} / \mathrm{m}^{2}$ and negligible compared to the osmotic pressure $P_{\gamma}$ of about $8 \times 10^{4} \mathrm{~N} / \mathrm{m}^{2}$ as estimated for a dilute solution with $33 \mathrm{Osm} / \mathrm{m}^{3}$.

[15] Y.-H. Li et al. (to be published). 